NASA Technical Memorandum 106332

AIAA-94-0993

\title{
NASA Lewis Meshed VSAT Workshop Meeting Summary
}

William Ivancic

Lewis Research Center

Cleveland, Ohio

Prepared for the

15th International Communications Satellite Systems Conference sponsored by the American Institute of Aeronautics and Astronautics

San Diego, California, February 28-March 3, 1994

(NASA-TM-106332) NASA LEWIS MESHED VSAT WORKSHOP MEETING SUMMARY

(NASA) $7 p$
N94-17487

Unclas 


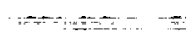 $\cdots=+\cdots$
$=\quad \because \quad-\quad-\quad-$ 


\title{
NASA LEWIS MESHED VSAT WORKSHOP-MEETING SUMMARY
}

\author{
William D. Ivancic \\ National Aeronautics and Space Administration \\ Lewis Research Center \\ Cleveland, Ohio 44135
}

\begin{abstract}
NASA Lewis Research Center's Space Electronics Division (SED) hosted a workshop to address specific topics related to future meshed very small-aperture terminal (VSAT) satellite communications networks. The ideas generated by this workshop will help to identify potential markets and focus technology development within the commercial satellite communications industry and NASA. The workshop resulted in recommendations concerning these principal points of interest: the window of opportunity for a meshed VSAT system; system availability; ground terminal antenna sizes; recommended multifrequency time-division-multiple access (TDMA) uplink; a packet switch design concept for narrowband channels; and fault tolerance design concepts. This report presents a summary of group presentations and discussion associated with the technological, economic, and operational issues of meshed VSAT architectures that utilize processing satellites.
\end{abstract}

\section{Introduction}

Since the mid-1980's, NASA Lewis Research Center's Space Electronics Division (SED) has been investigating satellite communications architectures that will use many of the technologies first developed for and tested on the Advanced Communications Technology Satellite (ACTS). Since 1989, SED has aggressively pursued studies and hardware development pertaining to meshed very small-aperture terminals (VSAT's). An area of recent interest has been the development of a switching and routing onboard processor for use in "thin-route" applications. The investigation into the development of a switch for meshed VSAT applications has raised several issues relating to networking, services, reliability, and economics. To gain insight into these issues and to disseminate NASA's concepts and concerns pertaining to them, SED committed to holding a meshed VSAT workshop.

The objective of the workshop was to address specific topics related to future meshed VSAT satellite communications networks including new services, switching architectures, uplink access techniques, ground terminal cost and complexity, and fault tolerance issues. The ideas resulting from this workshop will help to identify potential markets and focus technology development within the commercial satellite communications industry and NASA. Toward this end, SED invited participation from satellite service providers and systems suppliers; networking, switching, and routing specialists; and VSAT manufacturers.

The workshop, sponsored by NASA Lewis Research Center, was held June 2 and 3, 1992 in Cleveland, Ohio. Over 30 people participated with approximately half from industry or academia. The number of participants was intentionally limited to allow for effective communication and expression of ideas. On the morning of the first day, results of recent NASA-sponsored studies related to meshed VSAT systems were summarized. During the afternoon, participants, in groups of 6 to 12, discussed specific topics. Each group was given a list of issues and concerns to stimulate discussion. One group concentrated on services and protocol issues, one on ground terminal issues, and two others on onboard processing issues. The following morning, a representative from each group presented the group's findings and recommendations.

The following summarizes each group's presentation and ensuing discussion related to the technological, economic, and operational issues of meshed VSAT architectures that utilize processing satellites.

\section{$\underline{\text { Services and Protocols }}$}

The services and protocols group was asked to address as many of the following issues as time would permit:

(1) NASA has identified numerous services that could be enhanced by a processing and switching meshed VSAT network. What types of new and existing services does the industry predict will utilize a meshed VSAT network?

(2) NASA has been developing an architecture based on two discrete rates compatible with an integrated services digital network (ISDN): $64 \mathrm{kbps}$ and $2.048 \mathrm{Mbps}$. We are concerned that this may limit potential services. What range of data rates should be considered?

(3) Many existing communications scenarios require asymmetrical data flow (document retrieval, file transfer, and such). To what degree should this be addressed in the VSAT network? 
(4) Our present desire is to provide point-to-point, multicast, and full broadcast capability. What degree of multicast capability is envisioned?

(5) Design of the switching hardware depends heavily on the satellite throughput capacity. Studies indicate that, when using fiberoptic rings or similar shared bus architectures instead of other approaches, a break point occurs at approximately 2 to $3 \mathrm{Gbps}$. What throughput capacity is envisioned?

(6) To what extent will future meshed VSAT systems conform to protocol standards (e.g., ISDN, synchronous digital hierarchy (SDH), etc.)? Is it reasonable to assume that protocols can be absorbed by the ground station, or that meshed VSAT networks can be transparent to such protocols? What end-to-end, quality-ofservice/grade-of-service (QOS/GOS) requirements place the most severe constraints on future meshed VSAT networks? How will the need to interwork VSAT's into a terrestrial network affect design parameters?

(7) Rain fade is a problem at both $\mathrm{Ku}$-band and Ka-band; however, the fading problem is much more severe at Ka-band. What QOS and availability will be required in order to satisfy service users?

(8) What does industry see as the time-to-market or window of opportunity for meshed VSAT networks in general and, in particular, for meshed VSAT's that utilize onboard processing satellites?

(9) Studies show unfavorable satellite meshed very small-aperture terminal (M-VSAT) economics when compared with terrestrial alternatives for point-to-point traffic. This is partially because of the recent rapid inflation of space segment costs; but another significant factor, particularly for M-VSAT, is the EIRP (equivalent isotropic radiated power) burden of communication to small earth stations. What can be done to cut space segment costs per unit bandwidth serviced? For example, payload fractions of spacecraft typically account for 30 to 50 percent of on-orbit weight and a much smaller fraction of pre-perigee burn weight. Is there room for bus and propulsion technology advancements that would significantly increase this payload fraction? Another example: VSAT's are often defined as earth stations the size of about $1 \mathrm{~m}$ or less. Smaller size is assumed to have lower costs. Also, the earth station is less obtrusive and, therefore, would be less objectionable to surrounding communities and landlords. The penalty is this: cutting an antenna in half requires a quadrupling of transponder power to compensate. All other things taken to be equal, the spacecraft capacity would then be reduced by a factor of 4 and user costs would increase by a similar factor. Where user costs are dominated by the earth segment and earth station costs reduced by a factor of
4, then perhaps we have a wash. But do all these assumptions apply today? Is there still community and landlord resistance to earth station emplacements? In high-volume production, is earth station size a significant cost driver?

(10) NASA studies always assume Ka-band ground segment costs to be much higher than current VSAT costs. Part of this cost comes from the presumed complexity of Ka-band, and part from the wideband timedivision-multiplexed (TDM) operation at Ka-band. Are there fundamental limitations that would prevent large volume production of Ka-band earth stations at the same costs as C-band and Ku-band VSAT's?

(11) A major portion of the Ka-band EIRP burden is the power margin for rain fade alleviation. Part is raw power; part is large, heavy, high-gain multibeam antennas. For every $3 \mathrm{db}$ of rain margin, the satellite potential capacity is cut by half and user cost almost doubles. Are there ways to provide for rain fade on an intermediate basis ( 0.5 percent) without burdening the entire spacecraft with power margins that are rarely used? For example, a hybrid band S/C could be built where C-band is used in rain and Ka-band is used in clear sky. This is not a new idea. Japanese experiments demonstrated its technical feasibility and the idea was considered in early ACTS studies. However, it was judged impractical at that time because of excessive earth station costs and insufficient bandwidth at C-band. (At that time ACTS carried a significant trunking payload with more than $500 \mathrm{Mbps}$ going to New York City alone.) Would high-volume production of a dual-band earth station result in earth station costs significantly different than single-band earth stations costs? Now that trunks are carried by fiber, the amount of satellite traffic into highpopulation centers that would be at risk in a rain fade may be significantly less than that assumed in the ACTS studies. What would be a reasonable estimate of at-risk traffic $(1 / 5,1 / 10,1 / 100$, etc., of the original estimate)? Another example would make use of a single, high-power, wide-scan beam to compensate for rainfaded areas and all other beams designed for clear sky. Would schemes such as this unduly burden the spacecraft complexity and introduce unacceptable risks?

The services and protocols group was able to address most of the issues. The following is a summary of the group's findings and additional information conveyed during open discussions.

The services and protocols group felt that future meshed VSAT's will be required to handle data rates ranging from $64 \mathrm{kbps}$ through $8 \mathrm{Mbps}$. Potential applications will include point-to-point communication (e.g., voice), public safety networks, interactive video (e.g., training and distance learning), distributed 
cooperation (e.g., teleseminars, telemedicine, and general multipoint-to-multipoint applications), and all existing VSAT applications. As for the degree of multicasting required, the network will probably consist of a large number of small multicasters and a small number of large multicasters/broadcasters.

When considering services and protocols, many business considerations need to be addressed. The overall user cost must be comparable to existing systems. The existing VSAT applications must be "upwardly compatible." The system throughput should be as high as possible to allow for flexibility and expansion. The satellite hardware should be designed with as much capacity as is reasonable. The M-VSAT enables interenterprise connectivity and may be more practical and affordable to small businesses than standard "hub" VSAT's. Last, M-VSAT's will require system flexibility and compatibility, particularly for large enterprises.

The services and protocols group indicated that the window of opportunity for meshed VSAT's would be from 1996 to $2005 / 2010$ with only mobile applications remaining after this timeframe. To meet this window, systems should be demonstrated by 1994 and critical technologies should be in place by 1996. If meshed VSAT's are not available by 2005 , the infrastructure and investment will be used for terrestrial links; to then open this market to satellites will be extremely difficult.

Compatibility with terrestrial networks is considered essential, particularly with ISDN and broadband integrated services digital networks (B-ISDN); although for B-ISDN, compatibility may be at the protocol level and not necessarily at the high-rate compatibility level. Also cell relay compatibility is preferable to frame relay compatibility. Multidimensional service class parameters, such as reliability, delay, hop limit, and such, are needed, and these parameters may need to be dynamic. Compatibility is seen largely as a function of the earth station intelligence. The M-VSAT's must be able to provide nondegraded, end-to-end QOS, if required; however, a lower QOS may be provided at a lower cost.

From a system view, the M-VSAT will be required to provide minimally 99.9 percent availability; therefore, rain fade techniques, such as dynamic power control, rate adjustments, and network reconfiguration, must be developed. Antenna size up to $3 \mathrm{~m}$ is acceptable for business applications from both cost and regulatory (non-obtrusive) standpoints.

For Ka-band networks, a dual-frequency system may be practical. When considering production, specifically the cost of $\mathrm{Ka}$ - versus $\mathrm{Ku}$-band, Ka earth stations may not cost significantly more than Ku-band VSAT's. Also, Ka-band spot beams are problematic for multicasting and broadcasting because of the large number of spots needed to cover the continental United States.

\section{Ground Terminals}

The ground terminal group was given a list of issues and concerns that overlapped with both the services and protocols group and the onboard processing groups. The ground terminal group was asked to address issues (6), (9), (10), and (11) from the services and protocols group along with the following issues:

(1) What are the current and future cost drivers of a meshed VSAT ground terminal (radiofrequency (RF) equipment, installation, licensing, and the like)? What can be done to reduce these costs?

(2) The uplink access method heavily affects the uplink utilization efficiency and the satellite hardware implementation (demodulators, decoders, and switcher/ router). The present architecture is based on an FDMA uplink and TDM downlink. In relation to ground terminal costs and complexity, what is the optimum uplink access method for a meshed VSAT network (FDMA, TDMA (time-division-multiple access), CDMA (codedivision-multiple access), multifrequency TDMA, timeshared FDMA, etc.)?

(3) What are the advantages and disadvantages of synchronous and asynchronous uplink transmission in relation to ground terminal cost and complexity?

(4) A destination-directed packet switch appears to force the ground terminals to read all incoming transmissions and reformat the incoming packets. Is there an alternative to this high-processing requirement? Is this a reasonable requirement to place on a VSAT?

(5) What portions of the ground terminal are most vulnerable to failure? What level of fault tolerance and autonomy should be applied to the ground terminals?

(6) Previously suggested solutions to rain fade are added coding, reduction of transmission rate, power augmentation, and a combination of these. Are these solutions technically and economically practical?

The ground terminal group determined that the two main objectives of onboard processing for meshed VSAT's are these: to reduce the ground terminal RF cost and to improve interconnectivity. Therefore, only those functions that enable these objectives should be performed in the satellite. All other functions should be performed in the ground terminal to improve system reliability, reduce cost, and enable system upgrades. In addition, on-the-ground digital processing is cost effective. 
The main cost drivers for the ground terminals are the antennas, the RF equipment, the modem, and the digital processing functions, particularly the software development and the protocol converters. The highpower amplifiers are estimated at $\$ 1,000$ per watt for solid-state power amplifiers and should be able to deliver 10 to $20 \mathrm{~W}$ RF power. The cost driver in the low-noise receiver is the stable oscillator. Synchronization adds cost to the ground terminal, but is not considered significant. The antenna costs for $\mathrm{Ku}$-band antennas will remain constant. However, for $\mathrm{Ka}$-band antennas, the cost will increase as a result of the required improvement in surface tolerance. The antenna size will also remain in the 1.8- to $2-\mathrm{m}$ range since reduced antenna size not only decreases the gain, but increases the sidelobes. Therefore, the intersatellite interference for satellites spaced 2 to 3 degrees apart is increased.

For receive-packet processing of destination-directed packets, an upfront, dedicated packet processor running real time would be required to read and interpret all incoming information.

For rain fade compensation, two techniques could be applied: coding and power augmentation. Power augmentation is considered more costly, adding approximately $\$ 1,000$ per watt of additional standby power. Coding decreases the throughput capacity but is considered relatively inexpensive. However, coding offers extra performance advantages and does not require decoding onboard the spacecraft (although onboard decoding is beneficial).

The amount of fault tolerance required in the ground depends on the applications and user requirements. Maintenance cost is a function of ground terminal complexity and is significant. The following systems (in order of importance) should be protected or easily maintained: the HPA power supply, the receiver, the modems, the digital electronics, and the antenna.

\section{Onboard Processing}

The onboard processing (OBP) group was split into two subgroups to obtain more diversity of opinion. Each subgroup addressed the same issues and arrived at similar positions regarding OBP. The following issues were addressed:

(1) NASA has assumed that packet-switched data is the communication format of the future and is presently investigating a processing satellite that accommodates 64-kbps packets and 2.048-Mbps circuits using a combined circuit/packet switch. This combined switching system attempts to optimize the switch by eliminating overhead in the circuit switch while improving utilization of low-data-rate, low-throughput users in the packet switch. An integrated switch, in which all circuit data is packetized, would be less complex to implement but would increase bandwidth requirements for circuits because of packet overhead. Does the ease of implementation justify the increased inefficiencies due to increased overhead? Should future meshed VSAT networks support circuit switching only or packet switching only?

(2) Our present desire is to provide point-to-point, multicast, and full broadcast capability. What degree of multicast capability is practical?

(3) The uplink access method heavily affects the uplink utilization efficiency and the satellite hardware (demodulators, decoders, and switcher/router) implementation. The present architecture is based on an FDMA uplink and TDM downlink. In relation to onboard processing, what is the optimum uplink access method for a meshed VSAT network (FDMA, TDMA, CDMA, multifrequency TDMA, time-shared FDMA, etc.)?

(4) What are the advantages and disadvantages of synchronous and asynchronous uplink transmission in relation to satellite complexity and reliability.

(5) A destination-directed packet switch requires all source and destination information to be carried within each packet essentially "error free" to maintain acceptable quality-of-service. This information must be specific enough to indicate, at a minimum, the number of earth stations in the network, which can be quite large. Is this practical to implement?

(6) What areas of an onboard processing satellite are most vulnerable to failure and what level of fault tolerance and autonomy should be applied?

(7) Should NASA require fault tolerant design practices in technology development contracts? If so, to what degree?

The first OBP subgroup indicated that the satellite should be able to handle data rates ranging from a minimum of $64 \mathrm{kbps}$ to 2 to $4 \mathrm{Mbps}$ maximum with a multifrequency time-division-multiple access (MF-TDMA) uplink access scheme. Because MF-TDMA requires bit and frame synchronization, data rates greater than $4 \mathrm{Mbps}$ would cause excessive timing and synchronization problems and should be avoided. Bit synchronization would also make the demultiplexer simpler to implement. This subgroup felt that CDMA should be investigated as an option for low rate users.

The switching and routing should be designed as simply as possible to avoid overloading the OBP units and to maintain reliability. The switching and routing 
should be designed to handle multicasting and could be implemented as a destination-directed packet switch (DDPS), but only if all circuits were converted to packets on the ground (integrated switch). A DDPS (also known as a self-routing, fast packet switch) should be used onboard the satellite rather than a hybrid switch or a circuit switch to maintain maximum flexibility for future services. The hybrid switch was discarded because the future distribution of satellite circuit and packet traffic is unknown; therefore, both the circuit portion and the packet portion of the hybrid switch would have to be designed to handle the full capacity of satellite traffic. This would be a waste of satellite resources. Packet header construction is critical for this implementation. The congestion control method should also be as simple as it is practical. Fault tolerance should be at a chip or module level with cold (or perhaps warm) standbys, and the buses and control memory heavily protected.

The second OBP subgroup felt that the satellite should accommodate a variety of users and services including narrowband ISDN (64 kbps to $1.5 \mathrm{Mbps}$ ), multimedia services (voice, video, and data), broadcast educational services, and B-ISDN at $155 \mathrm{Mbps}$ and up. These high-speed links could also service supercomputer networking. Cross-links should be considered for domestic-to-international satellite links.

FDMA/TDM access was endorsed for circuitswitched data and MF-TDMA for variable rate and burst sources such as packet data. CDMA or hybrid MF-CDMA should be studied further for low-rate users with performance, OBP complexity, interference rejection, and co-channel rejection addressed. High-rate TDMA is not considered cost effective on the uplinks for low throughput users.

On the topic of synchronous versus asynchronous operation, the following observations were made. Synchronization of high data rate bursts adds cost to user terminals. By reducing the burst rate using MF-TDMA, the ground terminal cost can be reasonable. Synchronous FDMA can reduce power requirements onboard but will increase costs to user terminals and the network, whereas asynchronous FDMA can be reasonably implemented onboard. Synchronous CDMA produces greater capacity (number of channels) and CDMA has inherent synchronization capabilities; however, CDMA user terminals will be more complex than FDMA terminals.

This subgroup felt that a hybrid satellite supporting data rates from $64 \mathrm{kbps}$ to $155 \mathrm{Mbps}$ is the most likely implementation of OBP. Circuit switching would be utilized on the high-rate trunks and packet switching for low-rate users. A DDPS is practical to implement; however, great care must be taken to minimize the overhead. Overhead greater than 20 percent is considered excessive because it eats into the revenue production. Multicasting must be implemented for narrowband users but produces inefficient use of addressing and frames in a DDPS. A practical solution would be to have multicast group addresses to handle multicasting in the network control. This subgroup recommended that multicasting in a DDPS be studied further.

Fault tolerance should be designed in from the beginning. The OBP units should be protected at the chip level, not the system level. This subgroup felt that fault tolerance needs to be studied further, particularly to understand failure mechanisms. This subgroup also recommended that fault tolerant design practices should be addressed in technology development contracts but not implemented due to cost.

\section{Conclusions}

NASA Lewis' Meshed VSAT Workshop brought about much information that will help NASA focus its approach on development of future meshed VSAT architectures and identify the critical technologies that need to be pursued. Some of the workshop's more significant conclusions are as follows:

1. The window of opportunity for a meshed VSAT system is considered 1996 to $2005 / 2010$.

2. The system availability must be minimally 99.9 percent.

3. Ground terminal antenna sizes as large as $3 \mathrm{~m}$ would be acceptable for private business systems.

4. Multifrequency TDMA at 2 to $4 \mathrm{Mbps}$ is recommended for the uplink.

5. For narrowband ( $64 \mathrm{kbps}$ to $2 \mathrm{Mbps}$ ), the switch should be designed as a packet switch with circuit data reformatted into packets in the ground.

6. Fault tolerance should be designed in at the component level and fault tolerant design concepts should be considered in technology development contracts but not implemented because of excessive cost. 
Public reporting burden lor this collection $\alpha$ information is estimated to average t hour per response, including the time for reviewing instructions, searchling extsting data sources, gathering and maintaining the dala needed, and completing and reviewing the collection of information. Send comments regarding this burden estimate or any other aspect of this collection of information, including suggestions for reducing this burden, to Washington Headquaners Senvices, Dirbctorate lor Intormation Operations and Reports, 1215 Jefferson Davis Highway, Suite 1204, Arlington. VA $22202-402$, and to the Office of Management and Budget, Paperwork Reduction Project (0704-0188). Washington, DC 20503.

\begin{tabular}{|l|l|l|}
\hline 1. AGENCY USE ONLY (Leave blank) & $\begin{array}{r}\text { 2. REPOAT DATE } \\
\text { November } 1993\end{array}$ & $\begin{array}{r}\text { 3. REPORT TYPE AND DATES COVERED } \\
\text { Technical Memorandum }\end{array}$
\end{tabular}

\section{TITLE AND SUBTITLE}

NASA Lewis Meshed VSAT Workshop Meeting Summary

6. AUTHOR(S)

William Ivancic

\section{PERFORMING ORGANIZATION NAME(S) AND ADDRESS(ES)}

National Aeronautics and Space Administration

Lewis Research Center

Cleveland, Ohio 44135-3191

9. SPONSORING/MONITORING AGENCY NAME(S) AND ADDRESS(ES)

National Aeronautics and Space Administration

Washington, D.C. 20546-0001
5. FUNDING NUMBERS

WU-506-72-21

8. PERFORMING ORGANIZATION REPORT NUMBER

E-8093

10. SPONSORINGMONTTORING AGENCY REPORT NUMBER

NASA TM-106332

AIAA-94-0993

\section{SUPPLEMENTARY NOTES}

Prepared for the 15th International Communications Satellite Conference sponsored by the American Institute of Aeronautics and Astronautics, San Diego, California, February 28-March 3, 1994. Responsible person, William Ivancic, (216) 433-3494.

12a. DISTRIBUTIONAVAILABILTTY STATEMENT 12b. DISTRIBUTION CODE

Unclassified -Unlimited

Subject Category 17

\section{ABSTRACT (Maximum 200 words)}

NASA Lewis Research Center's Space Electronics Division (SED) hosted a workshop to address specific topics related to future meshed very small-aperture terminal (VSAT) satellite communications networks. The ideas generated by this workshop will help to identify potential markets and focus technology development within the commercial satellite communications industry and NASA. The workshop resulted in recommendations concerning these principal points of interest: the window of opportunity for a meshed VSAT system; system availability; ground terminal antenna sizes; recommended multifrequency for time-division-multiple access (TDMA) uplink; a packet switch design concept for narrowband; and fault tolerance design concepts. This report presents a summary of group presentations and discussion associated with the technological, economic, and operational issues of meshed VSAT architectures that utilize processing satellites.

\begin{tabular}{|c|c|}
\hline \multicolumn{2}{|l|}{ 14. SUBJECT TERMS } \\
\hline \multicolumn{2}{|c|}{ Meshed VSAT; Onboard processing; Protocols; Packet sw } \\
\hline $\begin{array}{l}\text { 17. SECURTY CLASSIFICATION } \\
\text { OF REPORT } \\
\text { Unclassified }\end{array}$ & $\begin{array}{l}\text { 18. SECURTY CLASSIFICATION } \\
\text { OF THIS PAGE } \\
\text { Unclassified }\end{array}$ \\
\hline
\end{tabular}

19. SECURITY CLASSIFICATION
OF ABSTRACT
Unclassified

